\title{
Drug Treatment of Disease
}

\section{DRUG TREATMENT OF GOUT}

BY

\author{
R. M. MASON, M.A., D.M., M.R.C.P. \\ Assistant Physician, Department of Physical Medicine and Regional Rheumatism Centre, the London Hospital
}

\section{Introduction}

Gout is even to-day one of the most satisfactory diseases for the physician to treat, for in doing so he can still exercise the art of medicine in the choice of therapy to meet the individual problem that each patient will present. He has a good variety of effective drugs to choose from, and their appropriate administration calls for some skill, while the severity of the pain in the acute paroxysm is such that he is likely to gain a grateful and appreciative patient. Moreover, he has an almost specific biochemical test-the estimation of the level of uric acid in the blood-both to support his diagnosis and to measure the effect of his therapy.

\section{Natural History}

It is axiomatic that all therapy must be viewed against the background of the natural history of the disease, but this applies with particular force in gout, since the stage of development of the disease process will largely determine the choice of drug. Moreover, a clear idea of the natural history will also enable a diagnosis to be made reasonably early in its course. When effective therapy is available, and this is the case with gout, it is highly desirable that the correct diagnosis is made early. The natural history of gout may conveniently be considered as occurring in four stages:

I. Asymptomatic Hyperuricaemia.-This precedes the first attack by a variable period and is likely to be recognized only by accident, or in the asymptomatic relatives of known gouty subjects. It may lead to error in those patients who by chance develop some other form of arthritis, since the presence of an inherited hyperuricaemia is little or no protection against the development of other disorders, including those of joints.

II. Acute Mon-articular Attacks.-Stage II begins with the first attack of arthritis. The patient may never pass beyond this stage, especially if the attacks begin in later life. Intervals between attacks may be as long as 50 years, and prolonged observation of the individual patient may be necessary to reveal the pattern of frequency of these attacks which punctuate periods of complete freedom from symptoms.

III. Poly-articular Attacks.-When multiple joints begin to be affected in individual attacks, the disease is usually characterized by more severe and prolonged episodes and thereafter-

IV. Chronic Gouty Arthritis develops, with progressive deposition of urate in joints, bone, and possibly elsewhere, especially in the kidneys.

The rate at which the disease is proceeding as well as the stage reached are both determining factors in deciding drug therapy. One patient may pass from Stage I to Stage IV in the course of a very few years, others may never in a lifetime pass beyond Stage II. A secondary factor determining drug therapy is the level of circulating uric acid. Opinions differ on the upper limit of normal, but as a working rule I have found the following figures reliable in practice:

Blood uric acid

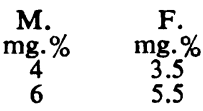

It is mandatory before making a firm diagnosis of gout to demonstrate that the patient has a blood or plasma level of uric acid above these figures. If this rule is neglected, mistakes in diagnosis are bound to occur.

\section{DRUGS AVALABLE}

Drugs available fall into three categories:

(1) Those which have either no, or an irrelevant, effect on excretion of uric acid but which are specific for the acute attack:

(a) Colchicine and its derivatives.

(b) Phenylbutazone (" butazolidin ").

(2) Drugs which cause an enhanced excretion of urate by the kidney but which have no immediate effect on acute gout:

(a) Salicylates.

(b) Probenecid (" benemid ").

(c) Phenylbutazone derivatives (G.28.315 or 4-phenylthioethyl-1,2-diphenyl-3,5-pyrazolidinedione).

(3) Non-specific anti-inflammatory drugs:

A.C.T.H., cortisone, and its variants-e.g., prednisone, prednisolone, triamcinolone, etc.

\section{Colchicine}

Colchicine is indicated in Stages II and III. It has maintained its place as one of the few specific drugs in medicine. Even though its use goes back some fifteen centuries, its mode of action remains obscure. It has no demonstrable effect on uric acid levels in the serum nor on urate excretion. It has the advantage that we have great experience in its use, and its toxic effects are well defined and easily recognizable. Although experimentally it is a mitotic poison, its clinical toxicity is characterized by gastro-intestinal disturbancediarrhoea or vomiting. The " diarrhoea dose " varies from one patient to the other, but usually remains constant for the individual. In the majority of cases the therapeutic dose is less than that of the diarrhoea dose. Colchicine is most conveniently taken in tablet form, colchicine B.P. gr. $1 / 120$ (0.5 mg.) -one every two hours. The tablets are entirely stable and the dose exactly calculated; they should be used in preference to mixtures. They may be carried in the waistcoat pocket, and every gouty patient being treated with colchicine should be able to produce his tablets on demand. Thus vital time is not lost in sending for the doctor or waiting for the chemist to open, and they can be taken at the first premonitory symptom-even, for example, irritability noticed by the wife.

If the diarrhoea dose is less than the therapeutic dose or if the patient is too impatient to await the effect of 
colchicine taken orally, it may be given intravenously. The dose is $3 \mathrm{mg}$., which should be made up in not less than $10 \mathrm{ml}$. (and preferably $20 \mathrm{ml}$.) of sterile water. Colchicine causes local irritation, and the greatest care must be taken to ensure that the needle is in the vein. The injection should be discontinued immediately if there is the slightest doubt of this. The injection can be repeated in 12 hours, the maximum dose in 24 hours being $6 \mathrm{mg}$. Intravenous colchicine does not usually produce disturbance of the gastro-intestinal tract. Demecolcine, deacetylmethylcolchicine ("colcemid "), has been mooted as an improvement on colchicine in that it produces less gastro-intestinal disturbance, thus enabling higher doses to be given. The initial dose can be as high as $4 \mathrm{mg}$., but alopecia, which may be complete, has occasionally been reported as a toxic effect, and since demecolcine is scarcely, if at all, better than colchicine itself there would seem to be no special indication for the use of this drug at present.

Colchicine can also be of use in interval therapythat is, between attacks to try to prevent their development. A dose of gr. $1 / 120(0.5 \mathrm{mg}$.) may be taken once, twice, or even thrice daily for a prolonged period, depending on the frequency and severity of attacks and the patient's tolerance, either alone or in combination with uricosuric drugs in Stages III and IV (see below). Prolonged intake of small doses of colchicine does not seem to reduce its efficacy in acute attacks.

\section{Phenylbutazone}

This now bids fair to replace colchicine as the drug of choice for the acute attack. It has the advantage that in most cases it causes no gastro-intestinal disturbance, especially when given for the short periods usually necessary. $200 \mathrm{mg}$. three times a day is normally sufficient, and many patients will respond to $400 \mathrm{mg}$. a day or less. Some prefer an initial single intramuscular injection of $1 \mathrm{~g}$. followed by oral administration.

Phenylbutazone is contraindicated only for patients with cardiac disease, peptic ulceration (past or present), hepatic or renal insufficiency, or a previous history of blood dyscrasia. The mode of action of phenylbutazone, like that of colchicine, in acute gout is not fully understood. Phenylbutazone also has a place in interval therapy, especially in Stage IV.

\section{Steroid Hormones}

I myself have never found it necessary to prescribe A.C.T.H. or cortisone, and its variants, in acute gout, though special circumstances might require their use. No special comment is necessary except to note that "rebound" attacks of gout are said to occur occasionally on their withdrawal.

\section{Salicylates}

\section{Uricosuric Drugs for Stages III and IV}

Salicylates have a uricosuric effect when given in adequate dosage, such as gr. 60-120 (4-8 g.) daily, reducing the uric acid level in the plasma to between 50 and $60 \%$ of pre-treatment values. Salicylism, if it occurs, usually does so early, and reduction of dose will relieve it. Subsequently the dose may be increased, often without recurrence. This form of therapy has the advantage of cheapness but the disadvantage that high dosage of salicylate must be maintained continuously. Few patients, in my experience, will accept this regime indefinitely, though some physicians have not found this difficulty.

\section{Probenecid}

While slightly less effective as a uricosuric agent than salicylates in full doses, probenecid has the advantage of ease of administration and relative lack of sideeffects. It is administered in $0.5-\mathrm{g}$. tablets, up to $2 \mathrm{~g}$. daily. Intolerance may occur, but it is usually mild and consists of an urticarial rash or nausea, occasionally dyspepsia. Renal colic or even urate stones may develop as a result of the enhanced excretion of urate, but adequate intake of fluids and making the urine alkaline will prevent this.

A curious phenomenon is the frequent occurrence of acute attacks during the early administration of this drug. Probenecid therapy should normally not be begun, therefore, during an acute attack, on which it has no direct therapeutic effect in any case, and the initial doses should be small-for example, $0.5 \mathrm{~g}$. daily for two weeks, $1 \mathrm{~g}$. daily for two weeks-and then a repeat estimation of serum uric acid should be carried out to assess the response. The dose can then be adjusted to keep the serum uric acid between 60 and $70 \%$ of pre-treatment levels. Colchicine should normally be administered prophylactically at the same time, a suitable dose being gr. $1 / 120(0.5 \mathrm{mg}$.) three times a day during the first three weeks, the dose thereafter being reduced to gr. $1 / 120(0.5 \mathrm{mg}$.) daily until there has been a prolonged period of freedom from attacks.

\section{Phenylbutazone Derivatives}

Two phenylbutazone derivatives have recently been developed-G.25.671 or 4-phenylthioethyl-1,2-diphenyl3,5-pyrazolidinedione and its sulfoxy metabolite G.28.315 or 4-(phenylthioethyl)-1,2-diphenyl-3,5-pyrazolidinedione. G.25 is probably too inclined to produce dyspepsia and G.28 appears to be the most promising drug. It is certainly the most effective uricosuric drug to date, being dose for dose some twelve times more potent than aspirin and some six times than probenecid. It can be given in the same doses as phenylbutazone.

It should be noted that the effect of both probenecid and of the phenylbutazone derivatives is much reduced by the administration of salicylates at the same time.

The foregoing provides a wide choice of drug therapy in gout. It is not possible to lay down hard and fast rules, but there are clearly two separate problems: (1) The treatment of acute attacks. (2) The prevention of acute attacks-that is, interval therapy. We cannot with our present knowledge prevent the patient from being a gouty subject. Therefore interval therapy means lifetime therapy. The decision to impose on a patient a lifetime of regular drug-taking, even of non-toxic drugs, is one which should not be undertaken lightly.

\section{Treatment of the Acute Attack}

Prompt administration of colchicine or phenylbutazone will control the acute attack of gout satisfactorily. Failure to do so requires a review of the diagnosis. Phenylbutazone, subject to the contraindications mentioned, is replacing colchicine as the drug of choice. Where the attacks of gout are infrequent this is all the treatment that is required.

\section{Interval Therapy}

Interval therapy should be considered :

(1) When the natural history of the individual disease appears to be rapidly developing-that is, when the 
patient has passed from Stage II through Stage III to Stage IV within a few years, especially if he is relatively young.

(2) When the acute attacks occur more than once a year or when they are particularly inconvenient-for example, in a barrister who may lose some important brief.

(3) When the serum uric acid is markedly raisedfor example, above $10 \mathrm{mg} . \%$. The critical level may be lower when there is a tendency to coronary artery disease, evidence of renal impairment, or attacks of thrombophlebitis.

(4) In chronic gouty arthritis when there is evidence of urate deposits in either joints or bone.

(5) When surgery is anticipated and a post-operative attack is likely.

Renal impairment is not a contraindication to uricosuric therapy; it is an indication for it.

Probenecid is the drug of choice, although the more potent uricosuric analogues of phenylbutazone may supplement this. Colchicine should be given in small doses, gr. $1 / 120$ (0.5 mg.) daily, at least during the first few months of administration and probably indefinitely. The effect of uricosuric therapy will be to reduce the frequency and severity of attacks, but this may not become apparent for some months, and in the early stages acute attacks may be precipitated. In the course of months and years, however, tophi will diminish in size owing to reabsorption of urate. Aspirin or salicylates should not be given at the same time as the uricosuric agent. Thus when there is a painful, chronic, gouty arthritis, long-term administration of phenylbutazone in a dose of $300 \mathrm{mg}$. a day may be justified, since this drug in addition to its effect on acute gout is a powerful analgesic and a mild uricosuric agent.

\section{New Appliances}

\section{ARTERIAL SUCTION ADAPTER}

Mr. R. E. HoRTon, F.R.C.S., Bristol Royal Infirmary, writes: The operation of embolectomy may fail not only because the tissues are dead as a result of delay in performing the operation, but also because of difficulty in clearing propagated thrombus which has formed in the stagnant blood column distal to the embolus. The artery can be regarded as clear only when there is a free flow of blood from the peripheral end of the arteriotomy. The adapter here illustrated permits the introduction of a length of polythene tube which can be used for clearance of a long length of artery through a single incision. Polythene tube has obvious advantages. It is flexible, does not collapse when suction is applied, and has a relatively large internal diameter. In one case, by its use both iliac arteries were cleared to the origin of the profunda femoris artery through a single incision in the aorta just above its bifurcation. In another case the whole of the femoral and popliteal arteries were occluded by thrombus secondary to an embolus at the origin of the profunda femoris artery. This was cleared with the aid of a second incision in Hunter's canal. The artery was finally cleared, and a brisk back-flow obtained when a second embolus lodged at the popliteal artery bifurcation was sucked out through the incision in Hunter's canal. In both of these cases the peripheral pulses were restored with most satisfactory results.

(Continued at foot of next column)

\section{To-day's Drugs}

With the help of expert contributors we publish below notes on a selection of drugs in current use.

Pipadone (Burroughs Wellcome).-This is dipipanone, or DL-6-piperidino-4 :4-diphenyl-heptan-3-one hydrochloride, an analgesic chemically related to methadone. It is supplied in ampoules, $25 \mathrm{mg}$. in $1 \mathrm{ml}$.

In a high proportion of cases, 20-25 mg. subcutaneously gives analgesia fully comparable to that of morphine. Nausea, sweating, headache, and some respiratory depression may be produced by high doses, but it is usually possible to achieve analgesia without these side-effects. Analgesia begins in 10 minutes, is maximum at 20 minutes, and persists for 5-6 hours. A few patients fail to respond. It is reported to have produced no constipation or anorexia, and no withdrawal symptoms, in a large series of patients treated for short periods. The possibility of addiction should be borne in mind.

Dipipanone is antagonized by nalorphine. It has slight atropine-like activity. The drug is metabolized by the liver, and should be given with special caution to patients with disease of liver or kidney.

Because of its relative freedom from hypnotic action, dipipanone is particularly useful for the relief of postoperative pain when immobility is to be avoided, and for inducing analgesia in obstetrics, when it may be used in place of pethidine. It may also replace pethidine as an adjunct to nitrous oxide in surgical anaesthesia, and is said to have less hypotensive effect than pethidine.

N.H.S. basic price : 12 25-mg. ampoules, 7s. $10 \frac{1}{2} \mathrm{~d}$.

\section{(Continued from preceding column)}

The adapter, which is manufactured by Chas. F. Thackray (Leeds), is based on the well-known principle of the polydapter which was described by Henderson (Lancet, 1950, 2, 291). It consists of two parts which screw together. As longitudinal pressure is exerted on the rubber collar it expands laterally, giving an airtight connexion on the polythene tube. Three adapters are available to take three sizes of polythene tube. The outside diameters of the polythene tube are $3 \mathrm{~mm}$., $4.5 \mathrm{~mm}$., and $6.8 \mathrm{~mm}$. Any length of polythene may be used, but 18 in. $(45 \mathrm{~cm}$.) has been found suitable.

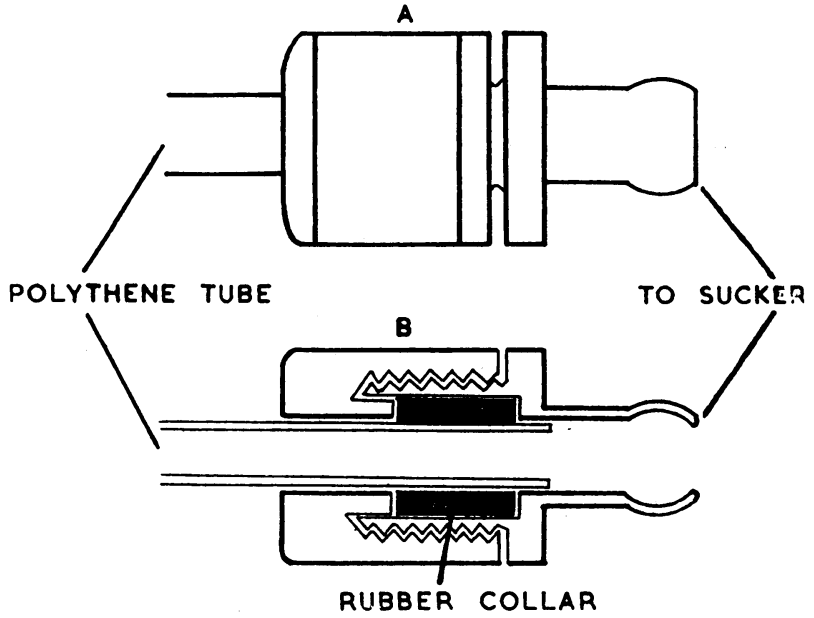

Two precautions need to be mentioned. One is that the end of the polythene tube which is introduced into the artery should be ground to a smooth surface to avoid trauma to the arterial wall; the other is that the adapter and polythene tube should be boiled separately to avoid damage to the polythene tube, and should be assembled after cooling. 